\title{
formación de sales eflorescentes durante la cockión
}

\author{
E. SCHMIDT - Instituto de Investigaciones Cerámicas de Essen \\ Die Ziegelindustrie, 20, n. ${ }^{\circ} 13$, julio 1967 , págs. $451-459$
}

\section{INTRODUCCION}

Un artículo sobre eflorescencias que apareció en 1965 en la revista "L’Industrie Céramique" (1) empieza con las palabras siguientes:

"El origen de las eflorescencias y la forma de impedirlas, así como la posibilidad de luchar contra la destrucción, son los temas principales de investigación de los expertos en la industria ladrillera."

Aunque esta afirmación es un tanto exagerada, hay que considerarla hasta cierto punto, ya que los efectos de las eflorescencias, del clima y de la helada no están resueltos de un modo claro y se les puede considerar como especialmente difíciles.

El autor citado describe una de las causas diciendo:

"Las dificultades en esta rama de la cerámica son debidas a la diversidad de materias primas que se emplean, lo que prohibe generalizar la solución encontrada en una empresa determinada."

Es verdad que este hecho impide particularmente la solución lo mismo que la investigación de este problema. A pesar de ello puede reducirse esta afirmación considerablemente, ya que existe por lo menos un punto que es válido para eflorescencias en cuanto provienen del ladrillo y, por regla general, también para los efectos de la helada (2) en todos los casos. Consiste en la aplicación de una temperatura lo más elevada posible, durante mucho tiempo y que sea siempre lá misma.

Con este trabajo se trata de informar sobre los complicados procesos de formación y deformación de sales eflorescentes durante la cocción. Estas tienen especial importancia en aquellas materias primas que forman compuestos salinos durante el proceso de cocción, es decir, que absorben compuestos de azufre del combustible. Se refiere principalmente a un grupo de materias primas para ladrillos que, por contener sustancias magnesianas y dolomíticas, tienden a la formación de sulfato de magnesio. Esta sal produce, cuando aparece en mayores cantidades, además de eflorescencias, daños en la superficie, los llamados "Abmehlungen" (= producción de harina). En la figura 1 puede verse un ejemplo típico. 
El informe se basa, aparte de las experiencias generales de la labor del Instituto y trabajos anteriores, en investigaciones realizadas en hornos de ladrillería completadas por experimentos en el laboratorio.

Fig. 1.-Enharinado por sulfato magnésico en una teja.

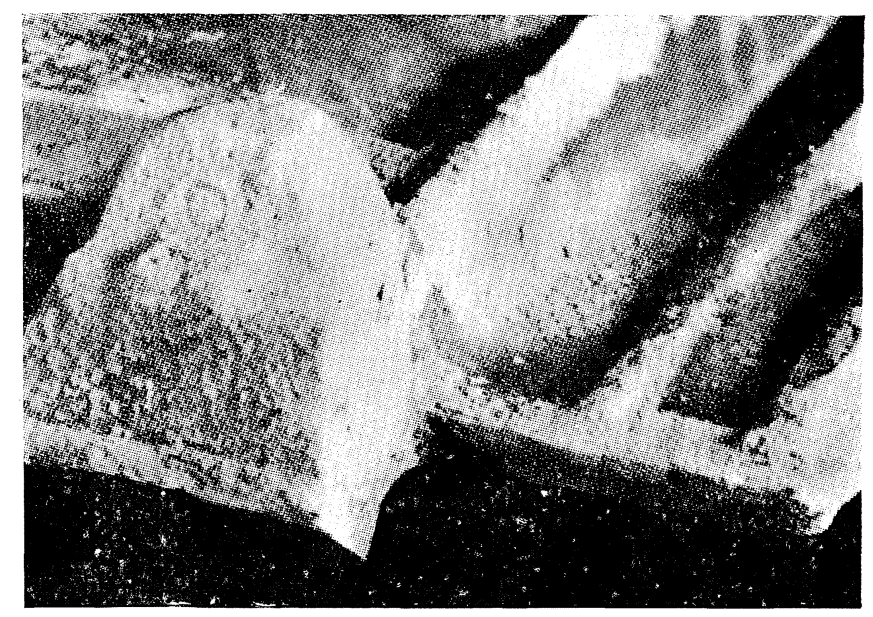

\section{COMPORTAMIENTo DE LOS COMPUESTOS DE AZUFRE DURANTE LA COCCION}

La finalidad de este trabajo era aclarar ciertos puntos en el comportamiento de los compuestos de azufre durante la cocción del ladrillo. Era de especial interés observar el ciclo del azufre que puede producirse dentro de un horno.

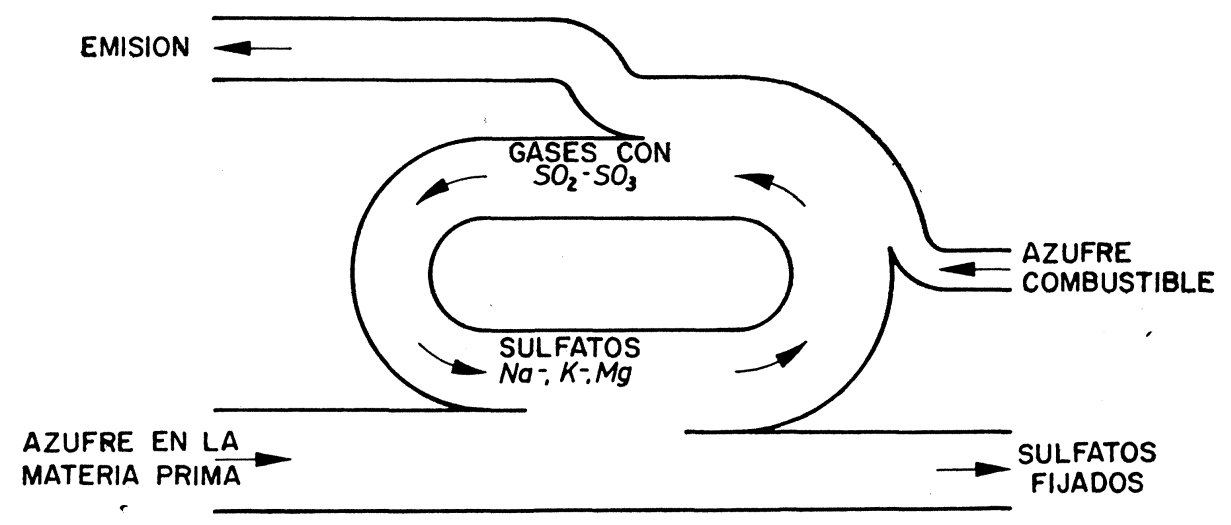

Fig. 2.-Ciclo del azufre (representación esquemática).

\subsection{El ciclo del azufre}

Con el gráfico de la figura 2 se trata de explicar lo que quiere decir el concepto de "ciclo del azufre". 
En todos los hornos continuos ordinarios, los gases del material y el aire se mueven en flujo opuesto. Visto del lado del material, el cual en el gráfico está representado por la parte horizontal inferior y moviéndose de izquierda a derecha, primero entra el azufre en el horno en la forma y cantidad que se encuentra en la materia prima (como pirita o como sulfato). En el lado opuesto, es decir, en el flujo de los gases de escape, se mueven compuestos de azufre en estado gaseoso, que provienen del azufre del combustible.

Ambas partes, durante el proceso de cocción, pueden modificarse considerablemente. Así, ya a temperaturas por encima de los $300^{\circ} \mathrm{C}$ empieza la descomposición de la pirita; con temperaturas mayores también se desccemponen los sulfatos, y a esto-además de la modificación del combustible-pueden unirse compuestos de azufre gaseosos en la corriente de los gases de escape. A la inversa, sin embargo, puede producirse otra vez una absorción de compuestos de azufre que empieza al fijar el gas $\mathrm{SO}_{2}-\mathrm{SO}_{3}$ sobre ciertos componentes de material, sobre todo compuestos de calcio, magnesio, potasio y sodio, creándose así sulfatos más o menos fijos.

De este modo se produce, y esto es válido para la mayoría de los hornos de ladrillo, una circulación interior de los compuestos de azufre, que en breves instantes se mueven del material a los gases de escape y viceversa.

Esta circulación no sólo tiene lugar en los hornos de ladrillo, sino también en otras instalaciones de cocción, en las que los gases de escape están en contacto con combustibles capaces de reaccionar. Dicha circulación se ha observado meticulosamente en los hornos de cemento. Sirve como ejemplo la figura 3, que procede de un trabajo de Sprung (3). (Hay que señalar que Sprung, además de la circulación del azufre, encontró una circulación análoga de los álcalis, que aquí no se va a tomar en consideración).

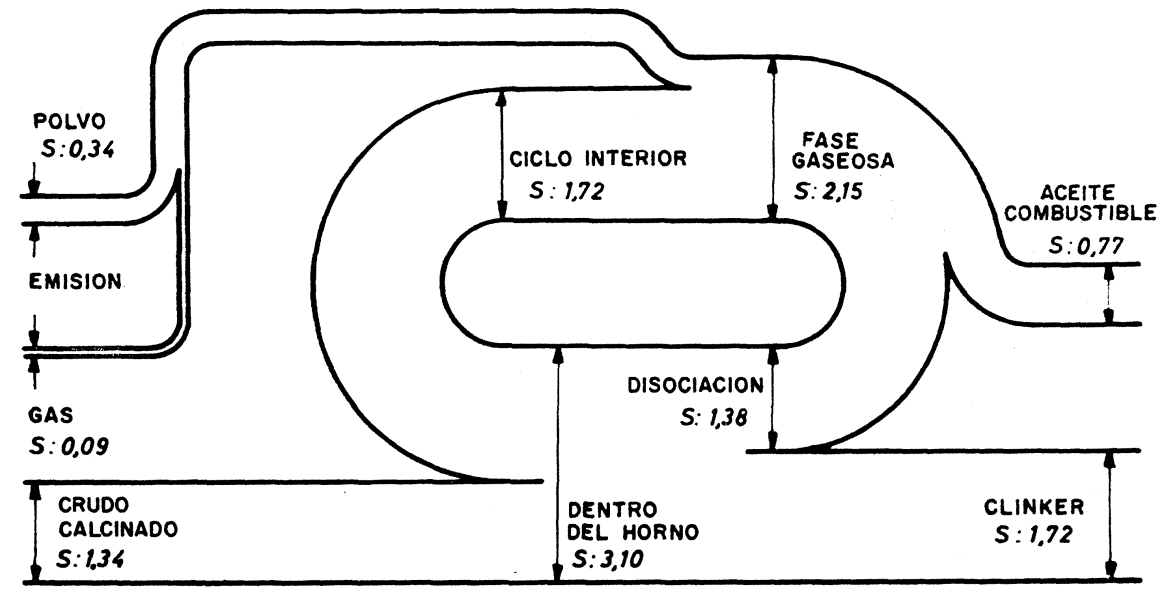

Fig. 3.-Ciclo del azufre en un horno de cemento (según Sprung).

\subsection{Normas de medida para comprobar la circulación del azufre}

Para conocer la circulación del azufre antes mencionada y las causas que la producen, se controla, por un lado, la forma del compuesto en cuestión en el material, y, por otro lado, la de los compuestos en los gases de escape. Mientras que para el primer examen pueden emplearse los métodos tradicionales, el otro, que hasta ahora constituía un pro- 
blema difícil, se hizo posible debido al desarrollo de métodos de medida de elementos que contaminan el aire.

Del trabajo en este sector proceden la mayor parte de los instrumentos modernos de registro empleados en este tipo de investigación. En la figura 4 se pueden observar los instrumentos más importantes para una medición de este tipo en un horno-túnel.

Fig. 4.-Aparato de medida de gases en un horno-túnel.

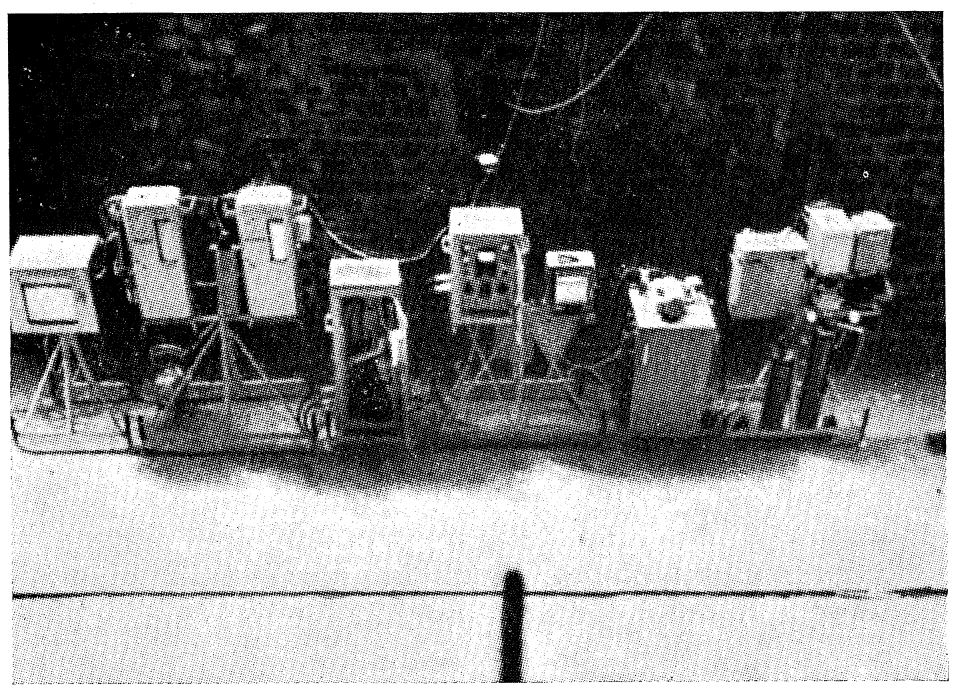

El instrumento más importante es el de la derecha, que sirve para la determinación continua y registro del $\mathrm{SO}_{2}$. Debido a que el agua resulta molesta en este instrumento, ya que trabaja según el principio de la absorción infrarroja, hay que congelarla previamente en el refrigerante contiguo.

Por primera vez pudo emplearse en esta investigación un instrumento de registro continuo (Sulfotherm de Siemens) para la medición de $\mathrm{SO}_{3}$, que está colocado en el centro del cuadro y que consiste en el analizador colocado abajo, el instrumento de dirección y el registro. En este instrumento se separa el $\mathrm{SO}_{3}$, o sea, el ácido sulfúrico que se ha formado de otros compuestos y se mide según el principio de conductibilidad.

Este instrumento se completa por 2 Monos para el análisis general de los gases de escape y por un registrador de compensación de 12 puntos para medir las temperaturas.

La disposición de todas las medidas puede verse en ia figura 5 .

Mediante un tubo de alúmina calentado en su parte superior se saca la muestra de gas por una de las toberas.

Una pieza sobrepuesta de cuarzo conduce el gas aspirado a los instrumentos de medida de $\mathrm{SO}_{3}, \mathrm{CO}_{2}$ y $\mathrm{O}_{2}$. El instrumento de $\mathrm{SO}_{2}$ se coloca sobre el refrigerador detrás del instrumento de $\mathrm{SO}_{3}$ y así utiliza el mismo conducto de gas. Justamente en el lugar de extracción, y mediante un termoelemento, se observan las temperaturas. Como los conductos calentados son bastante rígidos y requieren un calentamiento prolongado, esta disposición en la medida únicamente es posible en hornos de anillo o de zigzag, y en hornos túnel, pero sólo bajo ciertas condiciones, como, por ejemplo, instalando una abertura longitudinal continua. 
En las dos toberas laterales se introdujeron unas muestras, las cuales fueron sacadas después de determinados cambios de temperatura. Para controlarlas bien, al lado de estas muestras se instalaron unos termopares.

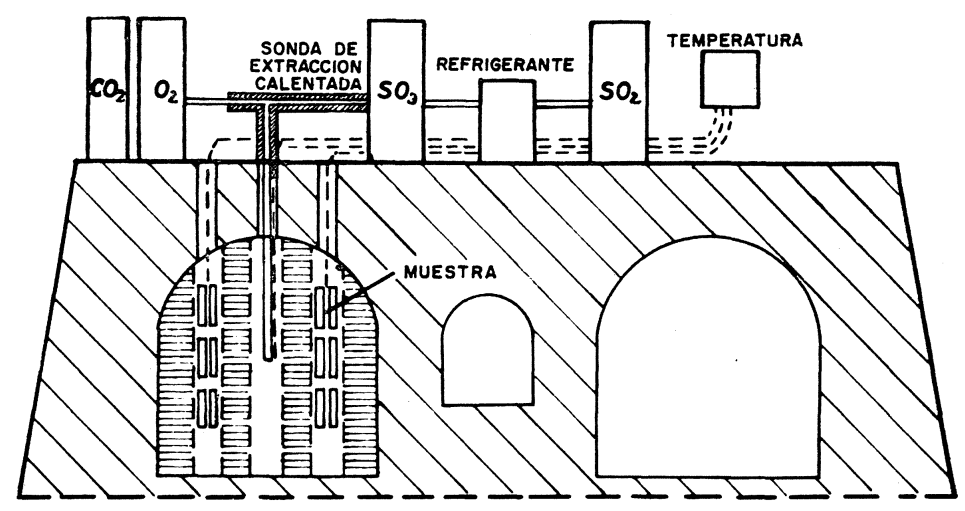

Fig. 5.-Esquema de colocación de los aparatos de medida para estudiar el ciclo del azufre.

Fig. 6.-Muestra para su colocación en el horno.

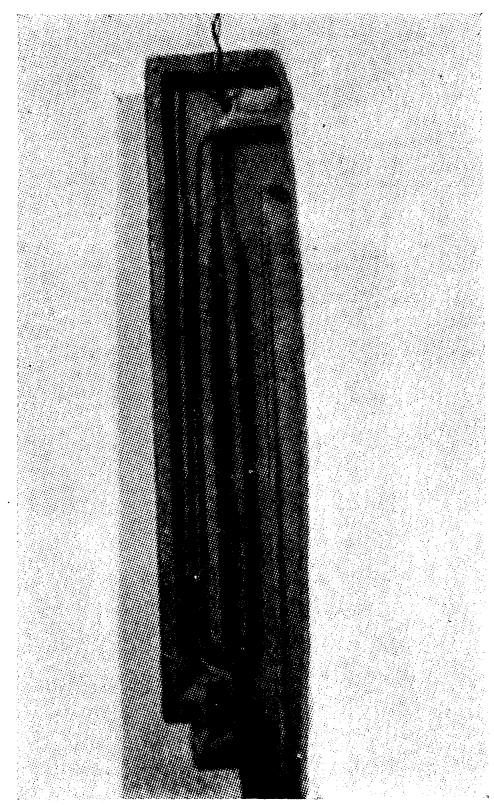

Las muestras de material (fig. 6) se cortaron de tal manera que siempre se incluían las secciones transversales más pronunciadas.

\subsection{Los resultados de una medición de horno}

Los resultados de una medición de horno llevada a cabo según el principio antes mencionado y referidos a la parte gaseosa, se encuentran en las figuras 7 y 8.

La figura 7 muestra las cantidades en el tiempo de los compuestos de azufre gaseo- 
sos que se produjeron al mover el fuego en el lugar de medición. Para la mejor orientación en el dibujo, se ha marcado la zona de fuego; la línea superior indica el curso de la temperatura.

Como se desprende de la curva de azufre total, la cantidad de compuestos sulfurados que pasan por el lugar de medición a partir de unos $500^{\circ} \mathrm{C}$ aumenta considerablemente y tiene una pendiente muy pronunciada poco antes de alcanzar la temperatura máxima. Hacia el final de la zona de cocción, la curva vuelve a descender mucho e, incluso, penetra en parte en la zona de enfriamiento.

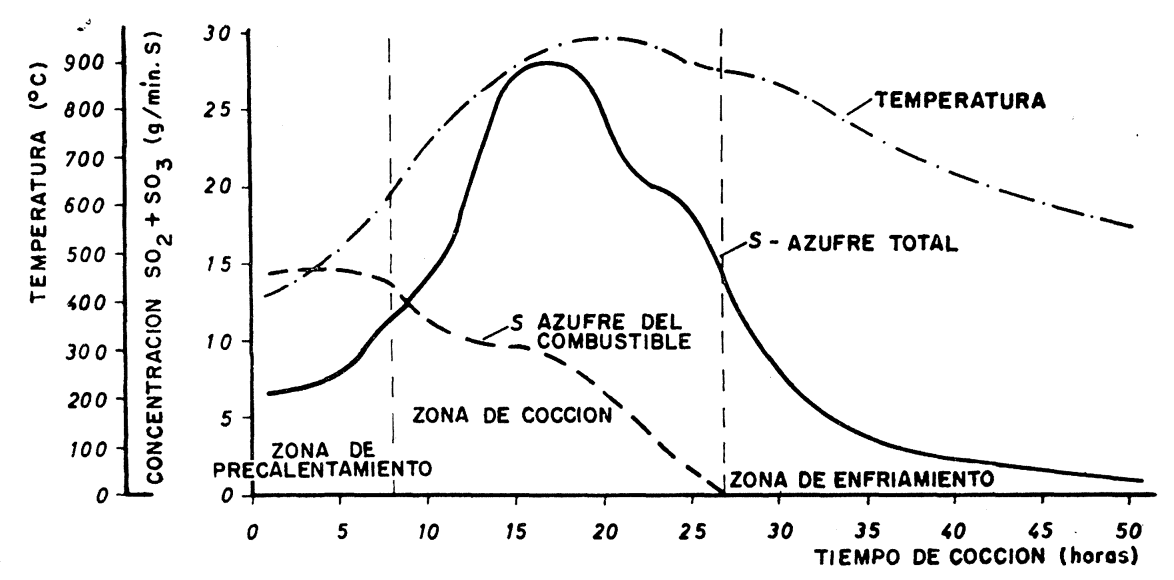

Fig. 7.-Cambios en la concentración de azufre en los gases de escape de un horno de tejas.

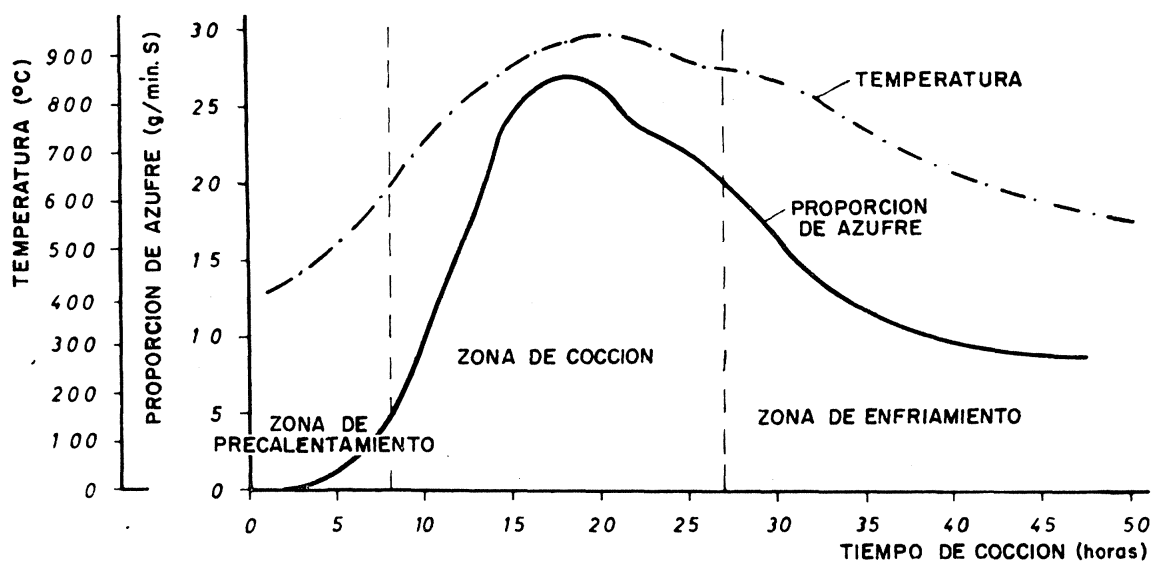

Fig. 8.-Cambios apreciados en la porción de azufre en el material a través de las medidas en los gases.

Para explicar dicha curva, vamos a observarla de derecha a izquierda, es decir, en la dirección del flujo de los gases de escape. Los gases de escape, y en primer lugar el aire refrigerante, atraviesan las distintas hileras de cocción de la zona de fuego y allí absorben continuamente los componentes de azufre del aceite combustible. Las cantidades que se incorporan en los gases de escape, se calculan por el consumo de combustible y figuran en la curva rayada. Se observa que la concentración máxima que se alcanza por este 
concepto sólo llega a la mitad aproximadamente del máximo de los valores medidos. Esta diferencia, lo mismo que la proporción que aparece en la zona de enfriamiento, es consecuencia de un desprendimiento de azufre procedente de la descomposición de sulfatos en el material. El descenso hacia la cabeza de fuego se debe exclusivamente a compuestos de azufre en el material con formación de sulfatos.

Estableciendo la diferencia entre las dos curvas, puede calcularse la aportación de componentes procedentes del material. Estos valores se encuentran en la figura 8.

La curva nos indica que en la primera fase del calentamiento tiene lugar una absorción fuerte de compuestos de azufre de los que, en gran parte, vuelven a descomponerse con un fuego más intenso. Al final, sin embargo, queda un sobrante de estos compuestos, con lo que el material, durante la cocción, ha experimentado un aumento en el porcentaje de compuestos de azufre.

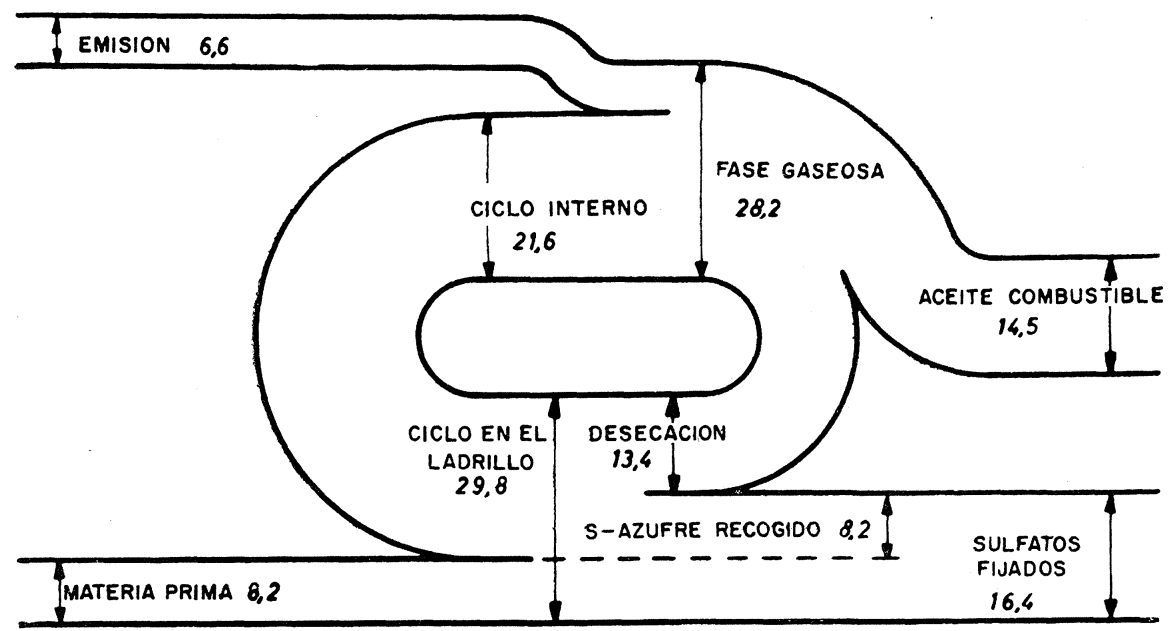

Fig. 9.- Ciclo del azufre en un horno de tejas.

Fig. 10.-Resultados analíticos de la muestra de material.

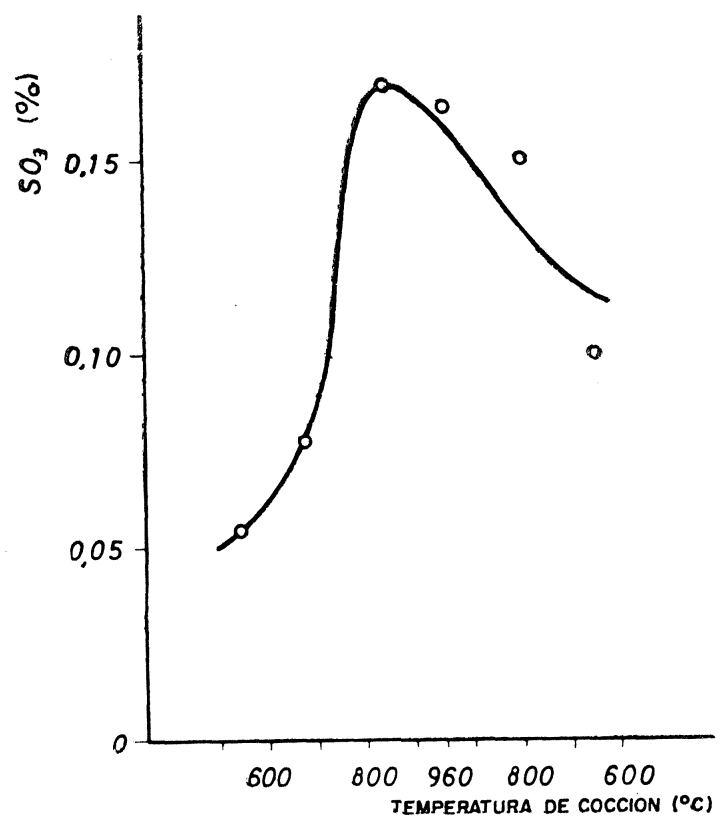


Mediante las dos figuras pueden calcularse también las cantidades que intervienen en el ciclo del azufre. El resultado se presenta de acuerdo con el ejemplo en que se muestra la circulación del azufre (fig. 9) al principio de este informe.

Una vez más se observa que el contenido relativamente reducido de azufre en el material aumenta considerablemente durante la cocción, mientras, por otro lado, la emisión de azufre en los gases de chimenea es más reducida que la introducida en el combustible.

Se ha comprobado la circulación del azufre por el examen de las muestras extraídas. Lo demuestra la figura 10, en la que se exponen los resultados numéricos del análisis de una serie de muestras.

Resultó difícil, no obstante, cubrir todo el campo de gases y material, ya que se produjeron grandes dispersiones entre las distintas muestras debidas, probablemente, a las diferencias en la elevación de la temperatura y en las condiciones del flujo.

\section{EXPERIENCIAS ADICIONALES DE LABORATORIO}

Del resultado de las mediciones de la circulación se deduce, para los productos en cuestión, el hecho de que sólo durante la cocción se forman sulfatos del azufre combustible. De aquí cabe preguntarse: ¿qué posibilidades existen para reducir el contenido en compuestos de azufre?

Después de lo dicho anteriormente es evidente que para este caso son indicados, en primer lugar, cambios de concentración de los compuestos de azufre en los gases de escape. Con este fin se hicieron experiencias en el laboratorio con pequeñas muestras para aclarar una serie de problemas relacionados con este asunto.

\subsection{Método de ensayo}

Para realizar las experiencias se montó una instalación, la cual permite la cocción de muestras cerámicas en una atmósfera controlada, y concretamente, en el caso que nos ocupa, en concentraciones variables de compuestos de azufre.

La figura 11 muestra la instalación experimental. De las botellas de gas (abajo a la izquierda) que contienen dióxido de azufre, nitrógeno y oxígeno, y por medio de rotámetros colocados en el armario de instrumentos, se conduce una mezcla definida de gases a un pequeño horno de catálisis, donde una cierta proporción de $\mathrm{SO}_{2}$ pasa a $\mathrm{SO}_{3}$. La mezcla modificada llega a un horno tubular que contiene la muestra y que por medio de un programa dirigido en el armario de instrumentos puede ser puesta en marcha de acuerdo con una curva definida de temperatura. Justamente detrás del horno, y a través de un conducto intermedio calentado, está conectado el instrumento de análisis para el $\mathrm{SO}_{3}$; desde aquí se conducen muestras de gas a otros instrumentos de medida que no se ven en la foto, para poder observar exactamente la concentración y sus modificaciones.

\subsection{Influencia de la concentración de azufre y de la relación $\mathrm{SO}_{2}-\mathrm{SO}_{3}$}

En las instalaciones descritas se trataron, en primer término, muestras de ladrillo en una atmósfera con concentraciones diferentes de $\mathrm{SO}_{2}$.

El resultado de estas experiencias se aprecia en la figura 12 y muestra un aumento notable de la absorción de azufre por el ladrillo a medida que aumenta la concentración de $\mathrm{SO}_{2}$. 
Era interesante, asimismo, si otros compuestos de azufre como el $\mathrm{SO}_{3}$, además de $\mathrm{SO}_{2}$, pueden influir la reacción con el ladrillo y en qué medida. Los ensayos con óxidos puros han demostrado, en parte, velocidades de reacción considerablemente más altas que con el $\mathrm{SO}_{2}$ como único compuesto de azufre en los gases.

En la figura 13 puede verse el resultado de un trabajo de Kuropka (4), relacionado con problemas de corrosión en materiales de construcción resistentes al fuego; este autor ha realizado experiencias con paso de gases a temperaturas bastante bajas. La representa-

Fig. 11.-Aparato de ensayos para la cocción de pequenas muestras en atmósfera controlada.
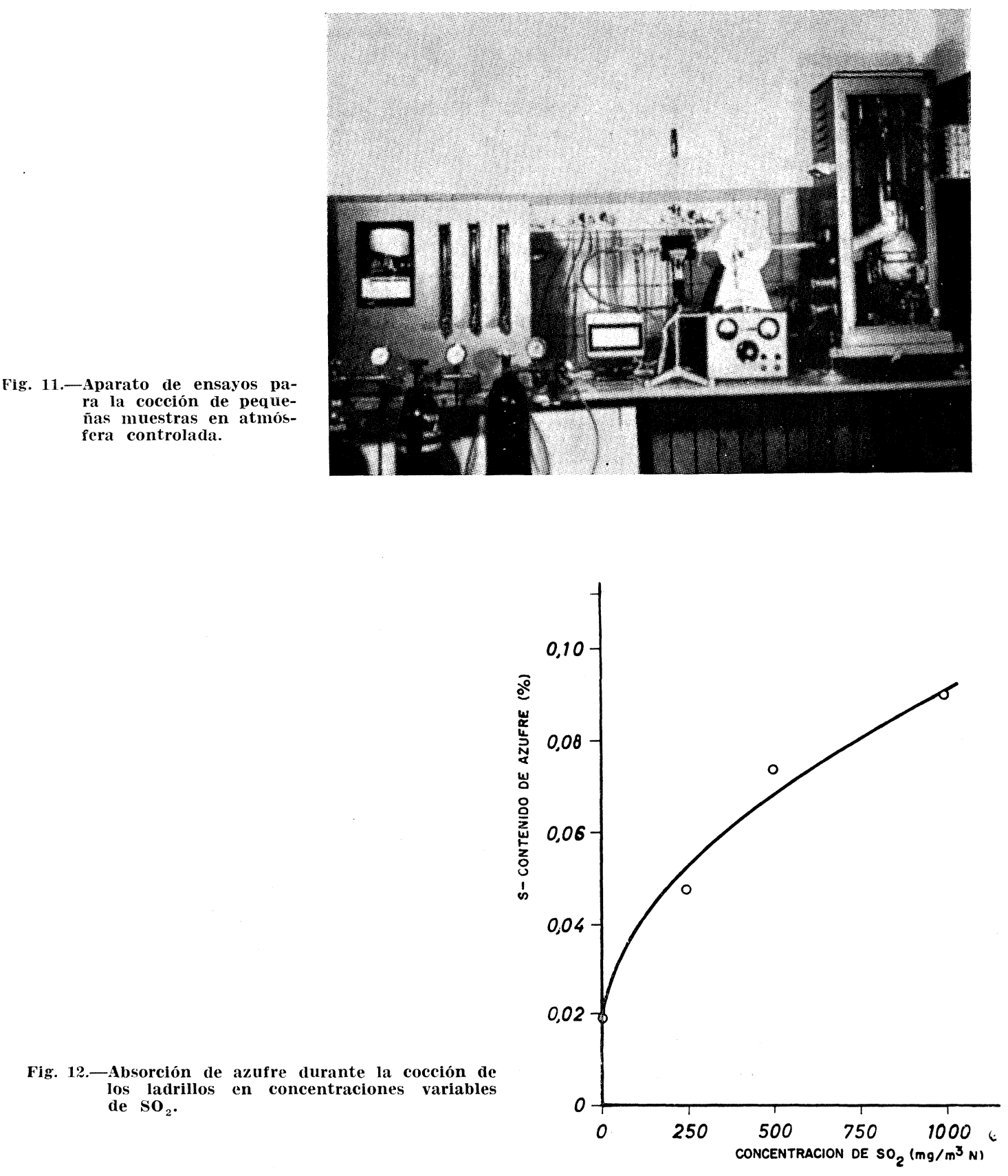
ción sencilla muestra que, sobre todo para el $\mathrm{MgO}$, la velocidad de reacción con el $\mathrm{SO}_{3}$ es mucho más alta que con el $\mathrm{SO}_{2}$.

Frente a los resultados de Kuropka, los experimentos de cocción del autor (fig. 14) sólo muestran pequeñas diferencias entre mezclas de gas que primero contenían $\mathrm{SO}_{2}$ puro, y luego $\mathrm{SO}_{2}$ y $\mathrm{SO}_{3}$ a partes iguales. En el tiempo total de la cocción del ladrillo parece que las variaciones de la relación $\mathrm{SO}_{2}-\mathrm{SO}_{3}$ tienen poca influencia en la velocidad de reacción. Por lo visto, parece decisiva la concentración total de los compuestos de azufre existentes. Sin embargo, queda en duda que transformaciones tienen lugar directamente en el sistema $\mathrm{SO}_{2}-\mathrm{SO}_{3}$ antes de la reacción.
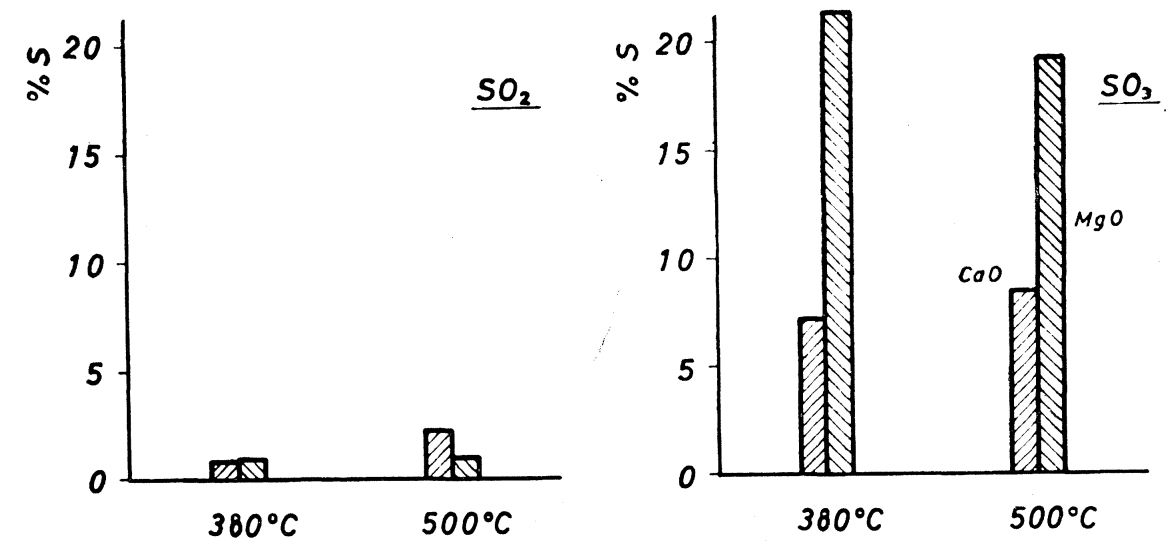

Fig. 13.-Absorción de azufre distinta, sometiendo los óxidos puros a $\mathrm{so}_{2}$ y $\mathrm{SO}_{3}$ gaseosos (según Kuropka).

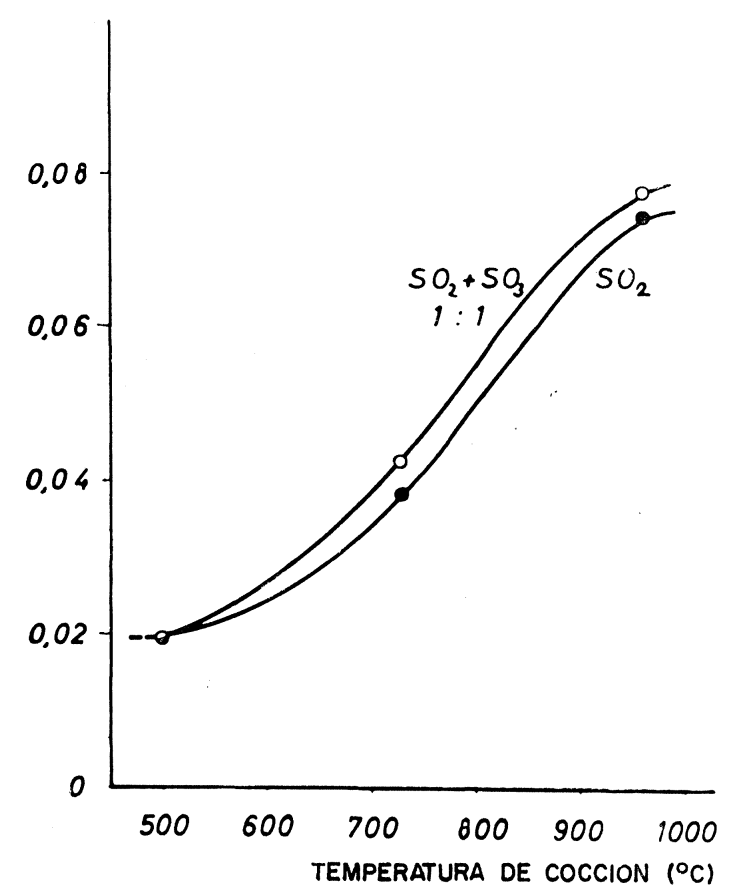

Wig. 14.-Absorción de azufre durante la cocción con $\mathrm{SO}_{2}$ y mezcla de $\mathrm{SO}_{2}-\mathrm{SO}_{3}$ puros.

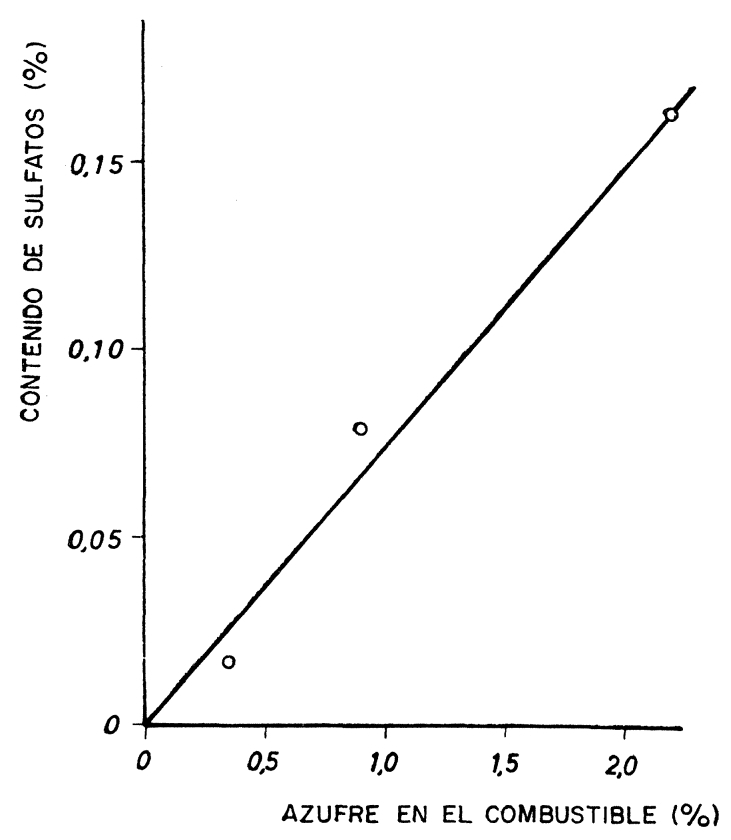

Fig. 15. - Absorción de azufre por cocción de ladrillos con combustible líquido y contenidos de azufre variables (sesún Rust). 
Una reducción de la absorción de azufre durante la cocción puede lograrse, pues, por una reducción de su concentración en el horno. Contribuye a ello, primordialmente, el empleo de un combustible que contenga menos azufre. De esto dio una prueba práctica Rust, quien observó la absorción de azufre por el ladrillo al emplear en varios hornos un aceite combustible con contenidos variables de azufre (5). En la figura 15, procedente de su publicación, se ve que en estos casos existe una relación casi lineal entre la absorción de azufre por ladrillo y su contenido en el combustible utilizado. Así se pone de relieve que una medida esencial para reducir los compuestos de azufre puede ser mediante el empleo de combustibles que contengan una proporción de azufre lo más reducida posible.

\subsection{Influencia del tiempo de cocción}

Mediante la curva de concentración de azufre mostrada en la figura 7 puede deducirse que es posible lograr una cierta reducción en la concentración de azufre con sólo prolongar la zona de fuego. Además, así, se prolonga también el tiempo de influencia en la fase de descomposición, lo que provoca una reducción adicional de los componentes de azufre.

Resulta, por tanto, que una duración prolongada del fuego a alta temperatura facilita la reducción de los compuestos de azufre en el material.

\section{COMPORTAMIENTO DE LAS SUSTANCIAS EFLORESCENTES DURANTE LA COCCION}

Las consideraciones hechas hasta ahora sólo se referían a la suma de los compuestos de azufre expresados como sulfato. Pero la tendencia efectiva al surgir la eflorescencia en los ladrillos no depende únicamente de la cantidad de dichos compuestos de azufre, sino mucho más de la forma en que se encuentran después de la cocción o que en el ladrillo terminado pueden recibir por influencia de la humedad o del clima.

\subsection{Solubilidad de los sulfatos}

Existe una diferencia muy importante entre el sulfato cálcico, por un lado, y los sulfatos sódico, potásico y magnésico, por otro. Esta se explica por la diferente solubilidad de los mismos, que se pone de relieve en la figura 16.

La poca solubilidad del sulfato cálcico tiene como consecuencia el que esta sal cree eflorescencias únicamente en casos excepcionales, como, por ejemplo, cuando aparece en grandes cantidades o con un contenido de humedad extraordinariamente elevado. En todos los demás casos no suele eflorescer.

Este hecho se manifiesta también al ver que los límites de eflorescencias mencionados en la literatura solamente se apoyan en los sulfatos que se disuelven fácilmente y que no mencioner el sulfato de calcio (6).

\subsection{El comportamiento de diferentes sulfatos durante la cocción}

De las solubilidades relativas puede llegarse a la conclusión de que, en general, para la cocción de Jadrillos sólo deben destruirse aquellas saies que se disueiven fácilmente, en tanto que el sulfato de calcio puede seguir existiendo.

Su relación con esto reside en el comportamiento de las distintas sales, ya que aqué- 
llas fácilmente solubles se descomponen o depositan a temperaturas bastante bajas, mientras que el sulfato de calcio es mucho más estable frente a la temperatura. La figura 17 muestra un ejemplo del distinto comportamiento de los compuestos solubles de álcalis y magnesio frente a los de calcio.

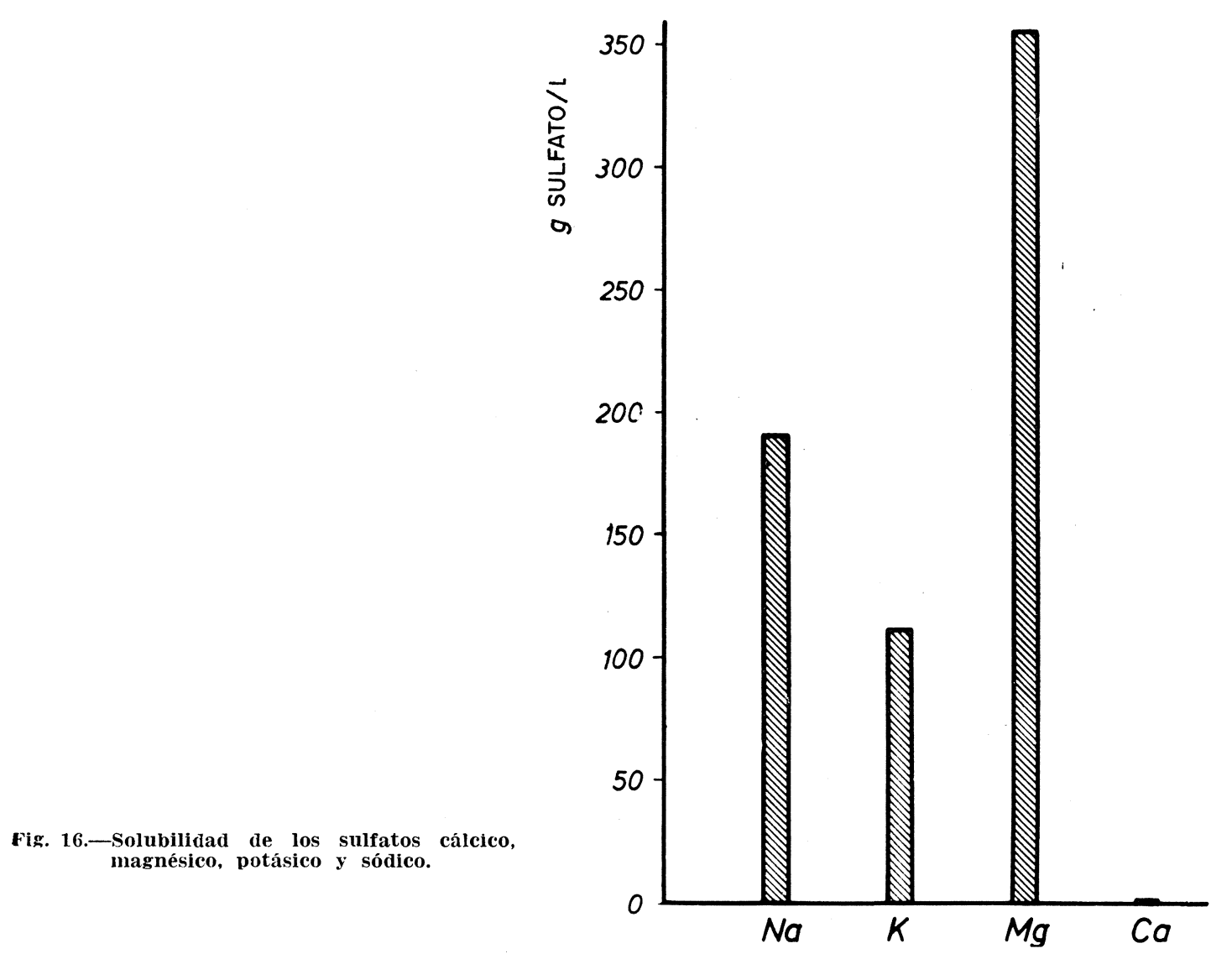

Los valores numéricos de estos análisis se han obtenido de las medidas realizadas en las muestras tomadas. Como se desprende del gráfico, la porción soluble de magnesio y álcalis alcanza un valor máximo a los $600^{\circ} \mathrm{C}$, pero a mayores temperaturas queda reducida a valores muy pequeños. Las combinaciones solubles de calcio, en cambio, aumentan hasta la zona de máxima temperatura. Así, bajo ias condiciones de máxima temperatura es pcsible destruir por completo las sustancias que se disuelven con facilidad. En un caso normal, por este procedimiento es posible eliminar por completo la tendencia a las eflorescencias.

\subsection{Transformación de sulfatos fácilmente solubles bajo la influencia de ácido carbónico}

Una circunstancia adicional que es preciso tener en cuenta en algunos casos, es la posibilidad de que el sulfato de calcio en el ladrillo fabricado puede ser transformado en sulfatos fácilmente solubles. El factor más importante en esta posibilidad proviene de una reacción creada por la actuación del ácido carbónico del aire:

$$
\mathrm{MgO}+\mathrm{CaSO}_{2}+\mathrm{CO}_{2} \rightarrow \mathrm{MgSO}_{4}+\mathrm{CaCO}_{3} .
$$


Esta reacción es la misma con los compuestos de sodio y potasio, de forma que es válida para todos los sulfatos fácilmente solubles mencionados.

La condición previa para la transformación del sulfato de calcio en sales fácilmente solubles es que los álcalis o los compuestos de magnesio en el ladrillo se encuentren en un estado tal, como por ejemplo MgO libre, que faciliten su disolución como consecuencia de esta reacción, aunque en ese estado no sean solubles en agua.

Este estado, sin embargo, depende, en gran medida, de la estructura mineralógica del ladrillo y, sobre todo, del grado de cocción; es decir, se le puede influir considerablemente por el tratamiento térmico.

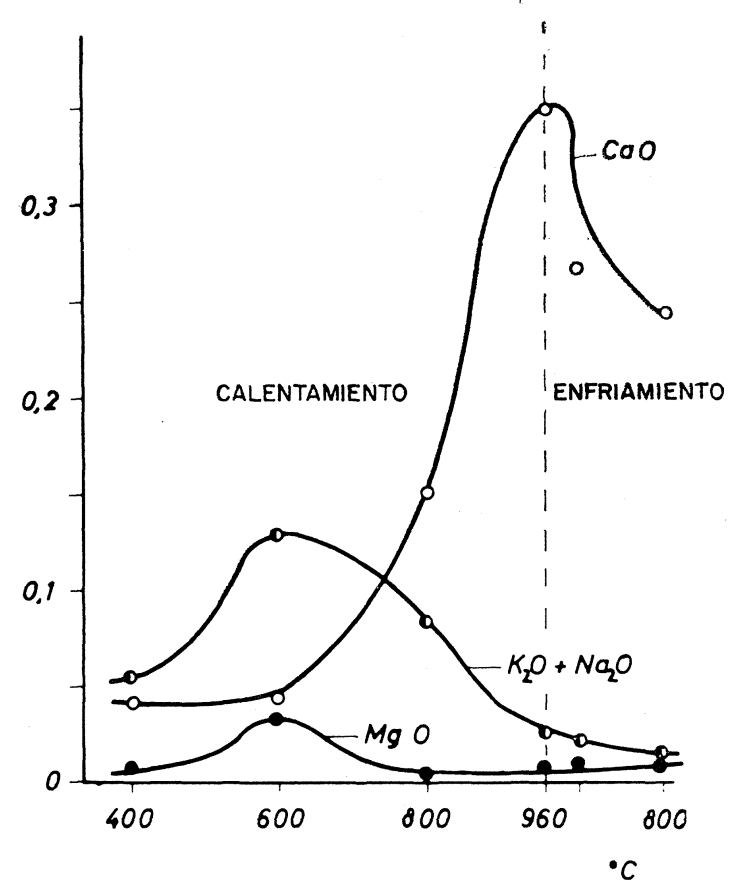

Fig. 17.-Variaciones de las porciones solubles durante la cocción.

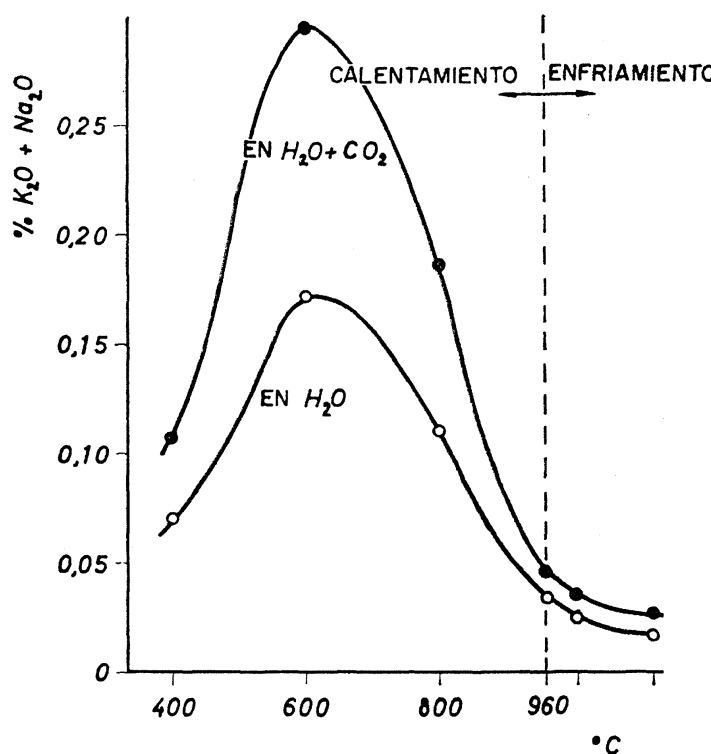

Fig. 18.-Variación de las porciones de álcalis solubles por el Co durante la cocción.

Resumiendo: se deben ampliar las exi gencias en la cocción de tal forma que, además de la destrucción de los compuestos fácilmente solubles, los compuestos de álcalis y magnesio deben fijarse en el ladrillo de manera que tampoco puedan disolverse por los procesos de reacción antes mencionados. Si esto no se consigue, una parte, o incluso el total del sulfato cálcico, puede transformarse en sales capaces de eflorescer.

Estos estudios se realizaron en las muestras ya mencionadas. En la figura 18 se ve el resultado del comportamiento de los álcalis.

El gráfico manifiesta que la fracción soluble por ácido carbónico es, en todas las temperaturas, notablemente más elevada que la fracción soluble en agua. Al llegar a la temperatura máxima, sin embargo, también en este caso se reduce a un valor numérico bastante bajo, de tal manera que en los ladrillos cocidos únicamente se conservan cantidades solubles en $\mathrm{CO}_{2}$ insignificantes. 
El comportamiento de las combinaciones de magnesio (fig. 19) se distingue notablemente del de las de los álcalis. Su fracción soluble en ácido carbónico en la cumbre de la curva es casi 100 veces mayor que la fracción soluble en agua.

Hay que añadir que para reconocer el máximo de la curva en la fracción soluble en agua, la escala en ordenadas se ha construido con los valores de las raíces cuadradas.

La diferencia más importante frente a los álcalis, no obstante, consiste en que la proporción de compuestos, en presencia de $\mathrm{CO}_{2}$, sea muy elevada a la temperatura máxima e, incluso, en el enfriamiento, sin sufrir una reducción considerable.

En la figura 20 se observan mejor los compuestos que permanecen en el ladrillo cocido; en el gráfico se representa el promedio de varios ladrillos, con el resultado de tres extracciones continuas. En la figura se representa además el contenido de $\mathrm{SO}_{3}$ (equivalente al $\mathrm{MgO}$ ). Se demuestra que hay una fracción de magnesio soluble superior a los sulfatos, lo que significa que, en este caso, al transcurrir el tiempo, todo el sulfato puede cambiarse en sulfato de magnesio.

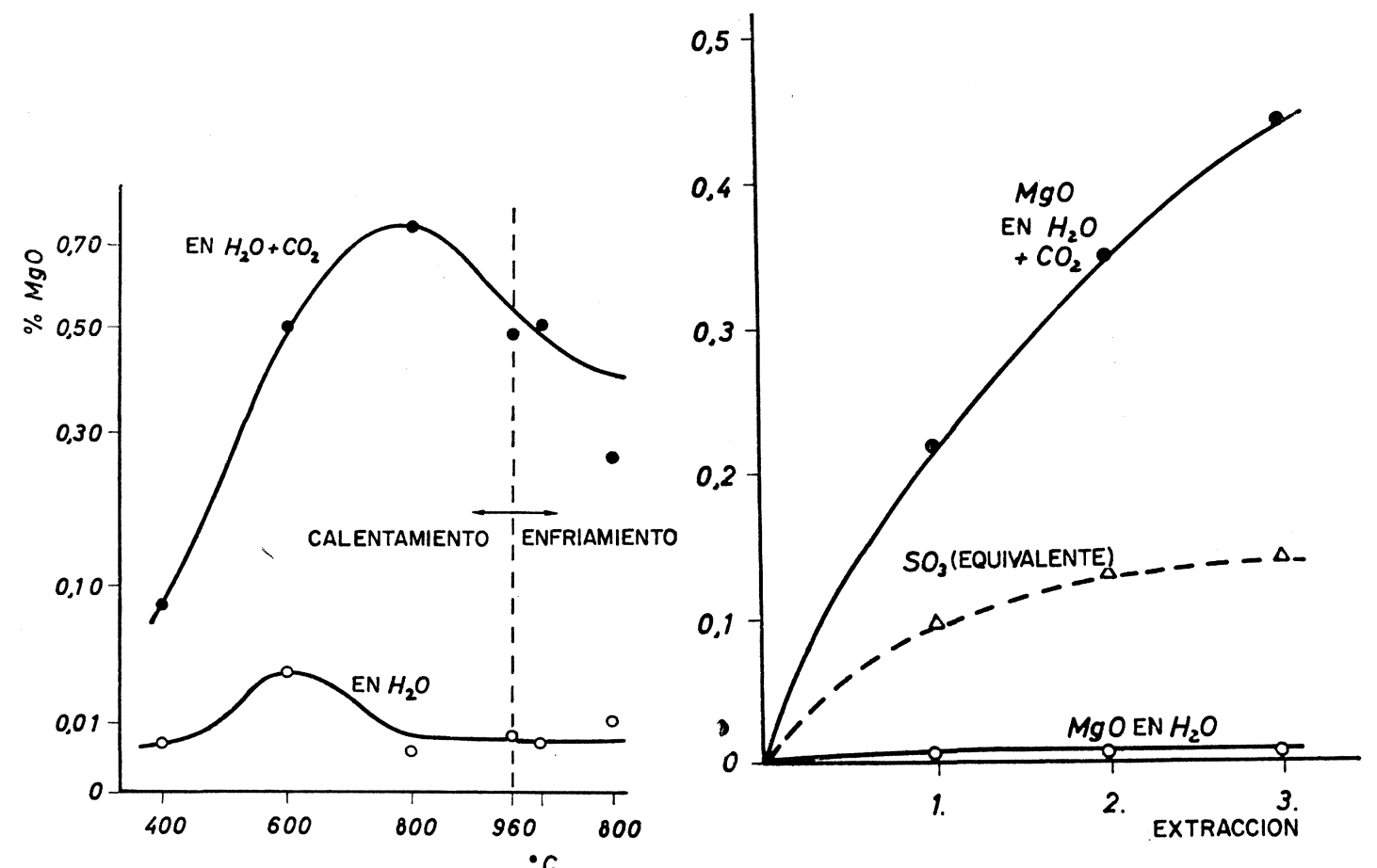

Fig. 19.-Variación de las porciones de magnesio solubles por el $\mathrm{CO}_{2}$ durante la Fig. 20.-Porción soluble en $\mathrm{CO}_{2}$ por extracción con-
tinua.

Hay que destacar, sin embargo, que las condiciones, según las observaciones realizadas hasta ahora en el Instituto, raras veces son tan desfavorables; los resultados de la práctica indican que los sulfatos no pueden aparecer más que en forma fácilmente soluble, con lo cual sólo tiene importancia la cantidad de sulfatos totales. 


\subsection{Modificación de los componentes solubles por el $\mathrm{CO}_{2}$ durante la cocción}

Después de los resultados descritos, es inmediato preguntarse cómo y con qué medios es posible una reducción de la fracción soluble de $\mathrm{MgO}$ por el $\mathrm{CO}_{2}$. Como solamente determinadas formas de las combinaciones de magnesio y de los álcalis pueden ser atacadas por el $\mathrm{CO}_{2}$, una reacción enérgica de los compuestos de magnesio con los componentes silicatados del ladrillo deben producir una mejora al formar silicatos estables. Para llegar a esto se piensa, en primer término, en una intensificación de los efectos de la cocción.

Para comprobar el resultado se cocieron varias de las muestras mencionadas por segunda vez en el horno del laboratorio, empleándose la temperatura máxima con tiempos de duración de 5,10 y 20 horas.

Como se desprende de los resultados que se encuentran en la figura 21, en todos los casos se logró una mejora considerable en la fijación de $\mathrm{MgO}$ al cabo de 5 horas de permanencia; temperaturas más altas aumentaron aún más la reacción. La cantidad de sulfato es mucho más reducida, de tal forma que la fracción total del mismo ya no tiene el papel más importante en la tendencia a la formación de eflorescencias.

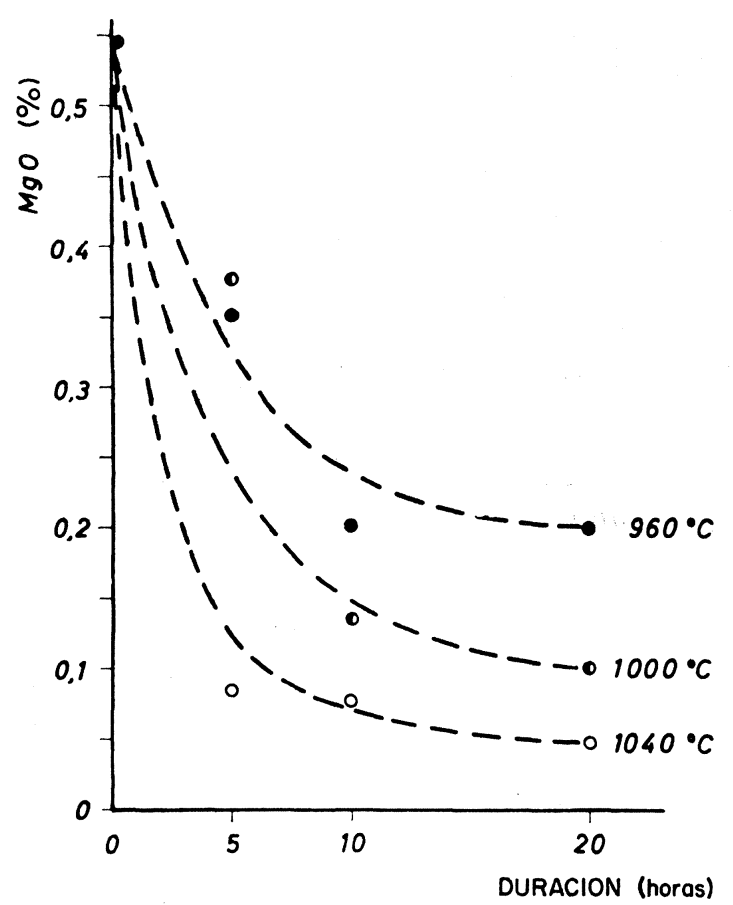

Fig. 21.-Reducción de la porción de Mgo soluble en $\mathrm{CO}_{2}$ al aumentar el tiempo de permanencia en el horno.

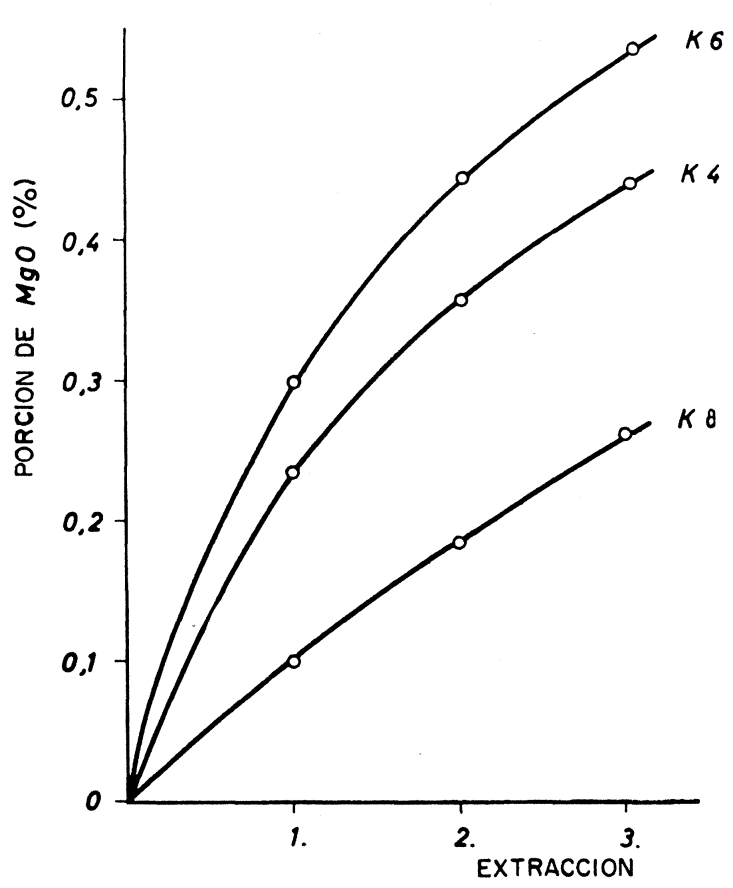
Fig. 22.-Porción de Mgo soluble en Co $_{2}$ de
diferentes muestras de ensayo.

La influencia de la temperatura también se hace notar en la cocción industrial, como puede reconocerse por las curvas de la figura 22. La extracción continua en varias muestras cocidas en distintas empresas indica diferencias muy notables en la fracción de $\mathrm{MgO}$ solubilizada por el $\mathrm{CO}_{2}$, hecho que únicamente puede explicarse por las diferentes temperaturas a que se expusieron dichas muestras. 
La figura da a conocer otra ventaja de la influencia de una temperatura alta y prolongada, y es que la fijación creciente de silicatos, además de reducir los componentes solubles, también disminuye la rapidez del ataque por el $\mathrm{CO}_{2}$. La reacción de los compuestos de calcio y magnesio, en fin, puede mejorarse asimismo por adiciones químicas y catalíticas. En uno de los pequeños experimentos realizados en el laboratorio, se añadió cloruro sódico al ladrillo de muestra recién formado; a pesar de las condiciones desfavorables de la cocción, es decir, rápido calenta miento y poca permanencia a alta temperatura, se produjo una reducción notable de la fracción de $\mathrm{MgO}$ soluble en el $\mathrm{CO}_{2}$.

En resumen: las últimas figuras demuestran que puede reducirse la tendencia a producirse eflorescencias, incluso en los casos desfavorables, no sólo por la reducción de sulfatos, sino también por medio de tiempos de permanencia en la máxima temperatura lo suficientemente largos y aplicación de temperaturas de cocción lo más altas posible. Adiciones que influyan de una forma químico-catalítica pueden aumentar tales efectos. Como en la práctica las condiciones, en la mayoría de los casos, son mucho más favorables, es decir, como las circunstancias previas que ofrece el material son favorables para la fijación de las fracciones solubles, una atención adecuada a la temperatura de cocción puede reducir las eflorescencias de manera satisfactoria.

\section{RESUMEN}

Por los resultados conseguidos por medidas en hornos y los experimentos y estudios realizados en el labolatorio, se trata de explicar los procesos que tienen lugar en la formación de los compuestos capaces de eflorescer durante la cocción.

Puede demostrarse que con las materias primas que tienden a absorber compuestos de azufre durante la cocción, la cantidad de azufre absorbido está en relación directa con la concentración de los compuestos de azufre gaseosos que se encuentran en el horno. Por este concepto, los compuestos sulfatados pueden reducirse empleando combustibles que contengan poco azufre, además de una mayor permanencia en el fuego.

Independientemente de esto, la tendencia a producir eflorescencias puede reducirse satisfactoriamente fijando tales compuestos sólidamente en el ladrillo. Esto se consigue con temperaturas de cocción lo más altas posibles y con tiempos de permanencia en la zona más caliente lo suficientemente largos. En ciertos casos se pueden intensificar las reacciones necesarias por adición de materias que actúen de manera químico-catalítica.

\section{bibliografia}

(1) Bodin, V.: “Un caso de eflorescencias”. L’Industrie Céramic, 1965, pág. 539.

(2) Piltz, G.: "Influencias de la fabricación en la resistencia de los ladrillos contra la helada". Die Ziegelindustrie 20 (1967), núm. 7, pág. 225.

(3) SPRung, S.: "El comportamiento del azufre durante la cocción del clínker de cemento". Tonind.-Ztg. 89 (1955), núm. 5/6, pág. 124.

(4) Kuropka, H.: "Efectos de corrosión de los gases que contienen dióxido de azufre en materiales de construcción inorgánicos entre $380^{\circ}$ y $500^{\circ} \mathrm{C}$. Brennst.-Wärme-Kraft 17 (1965), núm. 5, pág. 246.

(5) Rust, G.: "Experiencias sobre la formación de eflorescencias de sulfato magnésico en tejas". Die Ziegelindustrie 19 (1966), núm. 2, pág. 45.

(6) SpIngleR, K.: "La técnica del ladrillo". Halle: Knapp 1948. 